\title{
Le champ morphologique du mot phrase : approche historique et épistémologique
}

\author{
Sophie Jollin-Bertocchi ${ }^{1}$, et Jacques-Philippe Saint-Gerand ${ }^{2}$ \\ ${ }^{1}$ CHCSC (EA 2448), Université de Versailles St-Quentin-en-Yvelines (Université Paris-Saclay), France \\ ${ }^{2}$ Ex-UCA, 63380 Miremont, France
}

\begin{abstract}
Résumé. L'évolution du champ morphologique d'un mot-concept est significative du statut de ce concept et de son devenir. L'approche lexicale et lexicologique historicisée du métalangage est donc ici mise au service de l'étude d'une idée linguistique. De la rhétorique, qui configure le champ aux XVIIe et XVIIIe siècles, à la grammaire du XIXe siècle, où l'emploi de phrase se généralise, puis à la linguistique du XX $\mathrm{X}^{\mathrm{e}}$ siècle, phrase est un mot-morphème productif selon les principales voies de la création lexicale que sont l'emprunt, la dérivation suffixale et la composition populaire, qui se répartissent de manière cohérente selon les époques et les ancrages disciplinaires. Les définitions de la phrase attestées dans les dictionnaires depuis le XVIIe siècle emportent avec elles des connotations souvent négatives, que l'imagination onomasiologique a spécifiées dans la création de dérivés morphologiques. La linguistique a permis, au XXe et XXIe siècles, la conversion progressive de ces valeurs négatives en éléments d'une nouvelle terminologie intégrée à des cadres théoriques définis. La créativité lexicale, avec ses excès d'étiquetage, est alors adossée à un effort de théorisation sans précédent de la notion de phrase.
\end{abstract}

\begin{abstract}
The morphological field of the word sentence : historical and epistemological approach. The evolution of the morphological field of a word-concept is significant of the status of this concept. The historicized lexical and lexicological approach of metalanguage is thus here put at the service of the study of a linguistic idea. From rhetoric, which shaped the field in the seventeenth and eighteenth centuries, to the grammar of the nineteenth century, when the use of the word sentence became generalized, and then to the linguistics of the twentieth century, sentence is a productive word-morph according to the main paths of lexical creation, which are borrowing, suffix derivation and popular composition, which are coherently distributed according to the periods and disciplinary anchors. The definitions of the sentence attested in dictionaries since the seventeenth century often carry with them negative connotations, which the onomasiological imagination has specified in the creation of morphological derivatives. In the twentieth and twenty-first centuries, linguistics has made possible the gradual conversion of these negative values into elements of a new terminology integrated into defined theoretical frameworks. Lexical creativity, with its excess of labelling, was then backed by an unprecedented effort to theorize the notion of the sentence.
\end{abstract}

L'évolution de la notion de phrase depuis sa formalisation à la fin du XVIII ${ }^{\mathrm{e}}$ siècle a fait l'objet d'un certain nombre de travaux (Seguin 1993, Melis et Desmet 2000, Gautier 2006). Cette étude se propose de l'envisager au prisme de son champ morphologique. L'évolution du champ morphologique d'un mot-concept est en effet significative du statut de ce concept et de son devenir. C'est ce que cet article cherchera à vérifier à partir du mot phrase, l'approche lexicale et lexicologique historicisée du métalangage étant donc ici mise au service de l'étude d'une idée linguistique. De la rhétorique, qui configure le champ aux XVIIe et XVIIIe siècles, à la linguistique du XX $\mathrm{XX}^{\mathrm{e}}$ siècle, l'activité néologique est persistante. Après une mise en perspective des premières occurrences historiques du lexème phrase, et de la généralisation de son emploi dans la grammaire au XIXe siècle, on s'interrogera sur le lien entre le mode de formation des néologismes et la théorie dont ils participent, et sur le gain conceptuel de l'effort terminologique. Ces néologismes se comportent-ils comme de simples équivalents de leurs concurrents dans d'autres cadres épistémiques, ou apportent-ils une valeur ajoutée au plan conceptuel ? Selon un parcours chronologique, seront successivement envisagés l'innovation lexicale du XVIIe au XIX ${ }^{\mathrm{e}}$ siècle dans le champ de la rhétorique, puis les néologismes du $\mathrm{XX}^{\mathrm{e}}$ siècle dans la linguistique moderne.

\section{Onomasiologie et morphologie : du XVIle au XIXe siècle}

Comme le montrent notamment les travaux de Jean-Pierre Seguin (1993), de Valérie Raby (2002, 2008, 2018), de Nathalie Fournier (1998) et de Gerda Haßler (2012), y compris les remarques de Marc Wilmet (1997), les manuels de grammaire de la période allant de la seconde moitié du $\mathrm{XVI}^{\mathrm{e}}$ siècle au premier tiers du XIX ${ }^{\mathrm{e}}$ siècle ont un usage flou du mot phrase, qui interdit à ce dernier de se constituer en véritable métaterme. Récemment le collectif publié sous la direction de Franck Neveu (2019) montre également que cette notion, prise entre les concepts de proposition et d'énoncé, peut être envisagée de 
manière plurielle et ne se stabilise que progressivement à partir du XIXe siècle, lorsqu'elle est interrogée sous les angles de la philologie, de la grammaire et de son histoire, de la linguistique et même de la philosophie. À côté de ce foisonnement d'idées, qui n'est que l'expression naturelle du devenir de la pensée du langage, les travaux de lexicographie font figure d'apparents régulateurs et figent, à un moment donné, une doxa censée représenter et définir les usages - sinon le concept même - des mots dont ils dressent la nomenclature. Notre dessein n'est pas d'étudier une nouvelle fois la conceptualisation grammaticale ou linguistique de la phrase ; il est de s'attacher à une des caractéristiques les plus frappantes des usages de phrase que - précisément - n'enregistrent pas les ouvrages grammaticaux : sa productivité morphologique à des fins généralement terminologiques et axiologiques. C'est pour cette raison que nous nous emploierons à suivre les séries de dérivés morphologiques du mot phrase qui apparaissent dans quelques dictionnaires les plus remarquables, de l'époque classique au XIXe siècle.

\subsection{L'époque classique et la rhétorique}

La $1^{\text {ère }}$ édition du Dictionnaire de l'Académie (1694) consigne le sens du mot phrase:

Phrase Façon de parler. Assemblage de mots sous une certaine construction. Phrase ordinaire, populaire, phrase figurée, recherchée, bonne phrase, mauvaise phrase, phrase régulière, irrégulière.

Cette définition place dès l'origine la phrase sous l'hypothèque d'un balancement entre valeurs antagonistes (bonne vs mauvaise, etc.).

Dans cette même édition, les dérivés morphologiques de phrase sont des emprunts au latin, eux-mêmes issus du grec :

- Paraphrase, Paraphraste, Paraphraser portent en germe la dénonciation d'un excès de la verbosité d'une amplification rhétorique : " il faut dire les choses simplement, comme elles sont, sans les augmenter ».

- Antiphrase ; Épiphrase ; Métaphrase ; Périphrase, Périphraser, pour leur part, mettent l'accent sur la possibilité de détourner le sens de l'énoncé, ce qui implique l'existence d'un sens vrai exprimé par une « phrase » sans fioritures.

En réaction à la Préciosité ambiante de la seconde moitié du XVIIe siècle, un lien s'établit donc par l'intermédiaire de la rhétorique et de ses figures d'élocution entre le terme phrase et la notion d' « éloquence ».

\subsection{Le XVIIle siècle entre stabilité et évolution}

Le XVIIIe siècle a vu se développer, à partir de 1730, la querelle de la néologie, conforme aux modèles de la langue, opposée au néologisme, l'excès de la construction de mots nouveaux : « la néologie est un art, le néologisme est un abus » (Dictionnaire de l'Académie, 4' éd. 1762, 261 s. v.), à rapprocher d'une forme d'affectation et de préciosité du beau monde. La question se pose alors de savoir si les formes nouvelles que représentent les dérivés morphologiques de phrase relèvent de la néologie ou du néologisme. Sonia Vaupot (2012) a étudié avec précision la fortune des néologismes suffixaux créés par Louis-Sébastien Mercier. D'où il ressort que seuls s'inscrivent dans le lexique d'une langue les termes qui forment une entité conceptuelle répondant à un usage social défini par des conditions historiques. C'est ainsi que la $5^{\mathrm{e}}$ édition [1798] du Dictionnaire de l'Académie, revenant à la définition originelle de 1694, prend soin d'ajouter une entrée «Phrasier », à l'heure du Directoire et des Incroyables et des Merveilleus:

Phrasier. Faiseur de phrases. Il ne se dit que De ceux qui parlent ou écrivent d'une manière affectée et recherchée. Cet écrivain, cet homme n'est qu'un phrasier.

En 1761, le Dictionnaire grammatical de l'abbé Jean-François Féraud, mentionnait déjà :

Phrase, phrasier. Phrasier est un mot nouveau; il a l'air de faire fortune : mais il ne passera pas la conversation \& le style familier.

Tandis qu'en 1788, dans son Dictionaire critique, le même abbé Féraud proposait d'enregistrer l'usage momentané de phraseur comme manière d'exprimer l'agent, tout en notant que ce dernier terme était encore moins susceptible de s'inscrire dans la durée :

C'est un diseur, un faiseur de phrâses. Quelques auteurs ont dit phrasier en ce sens. On y proscrira l'étalage Des phrasiers, des rhéteurs boufis. [Gresset]

Le phrasier, le sec, l'amphigourique Sénèque. - M. Mallet du Pan dit, phraseur. Si l'usage admet l'un des deux, il est probable que ce sera le premier. [...]

Le suffixe -eur et le suffixe -ier sont extrêmement proches du point de vue sémantique puisque le premier est défini comme le «suffixe ordinaire des noms d'agents», tandis que le second désigne des personnes ayant " une activité en rapport avec la réalité désignée par le terme de base » (Grevisse-Goosse, 14 édition, $2008: 173$ et 175). Il est néanmoins possible de les départager au moyen de l'hypothèse que le suffixe -eur peut être associé à une valeur occasionnelle, circonstancielle, contrairement au suffixe -ier, qui dénote une valeur permanente. Cela permet peut-être d'expliquer la condamnation dont phraseur a été l'objet. Quoi qu'il en soit, les deux mots sont des termes mondains, et non des termes du métalangage.

À l'heure où se manifestent les premiers signes de la Révolution à venir et de ses bruyants discours, l'entrée de phrasier dans ces deux dictionnaires marque un malaise perçu. 


\subsection{Le XIXe siècle et l'assomption du Style}

La $6^{\mathrm{e}}$ édition du Dictionnaire de l'Académie (1835) se signale par une reconfiguration de la définition de phrase dans le sens d'unité grammaticale, tandis qu'il se généralise dans la grammaire, en accord avec l'évolution de la société et des mentalités. Le XIXe siècle développe en effet un effort de scolarisation (Guizot, 1833, Ferry, 1881) conduisant à la standardisation de la langue, et à une certaine représentation des ses qualités essentielles, dont témoigne la kyrielle des épithètes contraires susceptibles de la caractériser. Ainsi se construit un modèle du référenciel — grammatical et esthétique - de l'objet

PHRASE. s. f. Assemblage de mots construits ensemble, et formant un sens. Phrase ordinaire, populaire. Phrase figurée, recherchée. Bonne phrase. Mauvaise phrase. Phrase régulière, irrégulière, correcte, incorrecte, claire, obscure, bien construite, mal construite. Une phrase courte. Une phrase longue. Une phrase louche, embarrassée. Une phrase élégante, bien tournée. Faire une phrase. Construire une phrase. Prononcer, dire, composer, écrire, jeter sur le papier quelques phrases. La phrase grecque et la phrase latine admettent l'inversion. La phrase française a de la clarté.

Le Grand dictionnaire Universel du XIXe siècle de Pierre Larousse (1863 en fascicules, 1866-1876 en volumes) se signale par l'adjonction d'une entrée :

Phrasaire. Petit livre qui contient des phrases très courtes pour apprendre à lire aux enfants. Peu usité.

dans laquelle on retrouve l'effort pédagogique du siècle, et de l'auteur du dictionnaire en particulier.

L'entrée « Phrasier» du Dictionnaire de l'Académie (1835) apporte quelques légères mais significatives modifications par rapport à l'édition de 1798 :

Phrasier. Faiseur de phrases, celui qui parle ou qui écrit d'une manière affectée, recherchée, verbeuse et vide. Cet écrivain, cet homme n'est qu'un phrasier. Il est familier.

L'accentuation du sens péjoratif du terme dans la définition est encore renforcée par l'expression du marqueur de niveau de langue familier, qui implique un jugement dépréciatif, à l'heure où l'éloquence parlementaire, celle de la chaire, le journalisme, et la littérature font montre de leur pouvoir.

De même, contre l'avis précédemment signalé de l'abbé Féraud, phraseur fait son entrée dans la nomenclature de l'Académie :

Phraseur. Faiseur de phrases, celui qui parle ou qui écrit d'une manière affectée, recherchée, verbeuse et vide. Cet écrivain, cet homme n'est qu'un phraseur. Quel grand phraseur! Quel ennuyeux phraseur! Il est familier. On dit dans le même sens Phrasier.

En illustration de l'intérêt des Académiciens pour la langue telle qu'elle se pratique, la collocation "phrase faite " («faire rage, faire grâce, etc. ») retrouve la glose «Façon de parler» des éditions antérieures, à laquelle l'adjectif " particulière » et la référence à un usage consacré confèrent un statut phraséologique, sans que le mot ne soit prononcé, bien qu'il fasse partie de la nomenclature du dictionnaire.

Cette même $6^{\mathrm{e}}$ édition signe en effet l'entrée du terme phraséologie, lequel balance entre la langue et son usage littéraire :

Phraséologie. Construction de phrases particulières à une langue, ou propre à un écrivain. La phraséologie de la langue grecque, de la langue latine, de la langue française, etc. La phraséologie de cet auteur est bizarre, vicieuse.

Cependant, ni la $6^{\mathrm{e}}$ édition du Dictionnaire de l'Académie (1835), ni la suivante ne font une place au néologisme phraséologue, que Balzac avait introduit en 1831 et que Cormenin inscrit dans son Livre des Orateurs (1842), avec une caractérisation très précise :

Le Phraséologue ignore les lois et les affaires. Il n'a jamais ouvert le budget. Il dédaigne les chiffres, la logique, les faits communs et le train vulgaire des choses [...] Mais il est très-fort sur la mélopée ; il sait ce que c'est que l'onomatopée, le pléonasme, l'euphonie, la métonymie, l'hyperbole, la prosopopée, la protase, la catachrèse et autres figures de rhétorique à l'usage des Grecs. Il polit, il vernisse, il arrondit sa phrase dans le petit comme dans le grand, et il la fait reluire en bosse. Ce ne sont que fleurs, ornements, découpures et arabesques de style. [...] Les Phraséologues ne sont sensibles qu'à la musique du discours. Ils brodent sur tous les thèmes le chant de leur prose... [...] ils taillent leurs paroles, de même que le lapidaire taille les diamants à facettes. [...] Le Phraséologue ne se pique pas de raisonner. Il est vide d'idées, mais il est fourni de mots ; et il a étudié leur origine, leurs synonymes et leurs dérivés, dans les vingt-quatre lettres de l'alphabet. Il sait au bout du doigt le supin et le gérondif de chaque verbe. Il a scalpé la règle des participes et du que retranché. Son style est toujours en grande toilette; il le perle, il le dore, il l'habille à la dernière mode. C'est un fait de grammaire. [27-28]

Par l'involution du langage sur lui-même - phrase + logos —, cette caractérisation dénonce une sorte de tautologie dans les faits : à force de devenir une fin en soi la phrase détourne la grammaire de son objectif principal de standardisation de l'usage et l'oriente vers celui de la seule expressivité, qu'assume désormais la catégorie du style comme valeur.

Dans le Grand dictionnaire Universel du XIXe siècle de Pierre Larousse, la définition proprement lexicographique de Phraséologie reprend les sens classiquement reconnus, tandis que la partie encyclopédique, particulièrement développée, insiste dès le commencement sur les aspects négatif et ambigu de la chose : 
Encycl. Ce mot a signifié d'abord un recueil de locutions, mais la signification en a été depuis longtemps modifiée. On l'emploie aujourd'hui le plus souvent dans un sens défavorable comme nous le verrons plus loin. On l'a employé pendant des siècles et on l'emploie encore pour signifier la manière propre à une langue, ou particulière à un écrivain, de construire les mots et les phrases. On dit, par exemple, la phraséologie française, la phraséologie de Montaigne, celle de Fénelon, de Voltaire, de Chateaubriand.

Mais, très vite, il en vient à spécifier la marque distinctive de la phraséologie de la langue française :

[...] ce qui distingue principalement la phraséologie des langues anciennes de la phraséologie française,

c'est la fréquence et presque la continuité de l'inversion dans la prose aussi bien que dans les vers.

Dès que le terme phraséologie s'applique à l'ensemble des caractéristiques d'écriture d'un écrivain, il devient un parasynonyme de Style :

Tout le monde sait que la phraséologie de Voltaire se distingue par la clarté, la précision et la rapidité. Celle de Chateaubriand s'approprie aux traits plus que naturels de son talent, à se hardiesses au-dessus de la prose, et en même temps à l'éloquence émue et fière qui ressortait de son génie, aux caprices de l'imagination, aux mélancoliques rêveries qui remplissaient les esprits distingués de son époque. Les phraséologies diverses de ces quatre écrivains ${ }^{\mathrm{i}}$ nous montrent la langue française sous quatre aspects différents, qui en marquent les quatre phases importantes, au XVIe siècle d'abord, puis au XVIIe, au XVIIIe et au XIXe siècle. Mais, suivant la remarque de Fallot, les modifications n'ont guère porté en définitive que sur des points de détail ; quant à tout ce qui est fondamental et essentiel dans le langage, quant à l'esprit et à l'ensemble de la syntaxe, à la logique et au génie de la langue, l'identité est complète.

Quels que soient cependant les mérites singuliers des écrivains pour s'approprier l'essence d'une langue, le mot reste péjoratif :

[...] phraséologie se prend le plus souvent aujourd'hui dans un sens défavorable et signifie une manière

de parler vide et sonore, l'enflure de la forme ne recouvrant aucune idée sérieuse ou originale.

Et Larousse de rappeler que le journalisme et la réclame florissant au milieu du XIXe siècle étaient de sérieux promoteurs de cette phraséologie creuse.

Contemporain de Larousse, Littré est le premier à noter que le terme phraséologie pouvait trouver à se débarrasser « quelquefois » de sa connotation négative au profit d'un emploi technique en liaison avec le sens de sa base, mais qui est demeuré marginal :

$1^{\circ}$ L'étude et la connaissance de la phrase et, par conséquent, une partie très importante de la grammaire (peu usité en ce sens).

Enfin, les Académiciens de la $7^{\mathrm{e}}$ édition du Dictionnaire (1878), en dépit de l'évolution des connaissances linguistiques qu'impulsent les travaux de grammaire historique et comparée (Henri Weil, Auguste Brachet, Michel Bréal, etc.), se contentent de reprendre textuellement Littré en y ajoutant seulement deux exemples de leur cru, les dérivés suffixaux logue, -logie :

«Phraséologie ». « Il se dit quelquefois en mauvaise part pour signifier, Un discours creux, vide de sens.

Il nous fatigue avec sa phraséologie. Tout cela c'est de la phraséologie. ».

Les formes phraséologue et phraséologie, dont les dérivations suffixales rappellent le courant en voie d'extinction des Idéologues et de l'Idéologie, stigmatisent le défaut de la verbosité rhétorique et autorisent même, par un curieux retour en arrière, la redécouverte de phraséologiste $e^{\mathrm{ii}}$, pratiquant habituel de cet excès, dans la littérature journalistique de cette fin du XIXe siècle.

Depuis ses origines et jusqu'au XIXe siècle, l'Académie adopte donc une stratégie lexicographique assez ambiguë, puisqu'elle définit positivement l'objet «Phrase », tout en caractérisant négativement les dérivés morphologiques suffixaux auxquels il donne lieu, sans que l'on puisse décider précisément aujourd'hui si cette négativité est justifiée par la nature même de la suffixation, telle que les remarqueurs et les grammairiens l'envisagent et la discutent, ou seulement par un usage dûment enregistré filtré à l'étamine du goût.

En résumé, les néologismes du XVIIe siècle privilégient l'emprunt aux langues anciennes, alors que ceux du XVIIIe et du XIXe siècle reposent sur la dérivation suffixale, et se chargent de valeurs négatives. Les vassaux morphologiques du terme phrase se rattachent alors davantage aux catégories de la rhétorique, de l'esthétique de la langue (Alabalat 1899) et d'une éthique de la parole (Deschanel 1898) qu'à celles que la linguistique du XIXe siècle va faire progressivement émerger.

\section{La linguistique moderne et l'inquiétude terminologique}

Les linguistiques structurale, générative et phraséologique vont successivement faire évoluer la terminologie en fonction de leurs objectifs.

\subsection{Le structuralisme et la linguistique générative}

$\mathrm{Au}$ fur et à mesure de l'essor de la linguistique du XXe siècle, la désignation des unités de la langue et du discours requiert la création de nouvelles lexies. Le TLF met d'ailleurs en évidence la productivité du mot-morphème phrase en regroupant 
les néologismes dans un article au titre de «Formant - phrase, - phrastique ». À rebours des néologismes du XVIIe siècle, et dans le prolongement du XVIIIe et du XIXe siècle, ceux du XX ${ }^{\mathrm{e}}$ siècle continuent d'être forgés par dérivation suffixale, ce dont témoigne l'adjectif - et morphème - phrastique (E. Pichon 1933 dans R. Philol. fr. t.45, p. 69 ; cité dans le TLF), parallèlement à des créations fondées sur la composition populaire, dans le champ de la linguistique structuraliste et générative.

\subsubsection{Lucien Tesnière (1936) : mot-phrase et phrasillon}

Le terme mot-phrase n'apparaît pas dans la première édition de Grevisse (1936). Damourette et Pichon (1911-1927), qui emploient le composé sous-phrase, n'étaient sans doute pas loin de forger mot-phrase, dont ils se sont approchés en identifiant tout au moins le phénomène :

La vieille conception classique, et qui convient à l'immense majorité des phrases françaises, définit la phrase un ensemble gravitant autour d'un verbe. Mais il y a des phrases sans verbe, dites phrases nominales (...).

D'autres phrases sont réduites à un seul mot : c'est un appel (Jean.) ; une question (Quoi ?) ; une réponse réduite à sa plus grande simplicité $(\mathrm{Oui})$; une exclamation (Zut !) (69)

Tesnière, dans un article de 1936, emploie le néologisme mot-phrase, pour lequel il propose comme équivalent dérivationnel phrasillon, qui fonctionne comme une définition synonymique :

Dès que l'on essaye d'analyser les interjections pour voir dans quelle espèce de mots il y a lieu de les ranger, on s'aperçoit vite qu'elles sont au fond inanalysables, parce qu'elles renferment à elles seules le contenu sémantique de phrases entières. Elles débordent toute tentative de classification en espèces de mots, parce que ce ne sont pas des espèces de mots, mais des espèces de phrases. Nous les appellerons des mots-phrases, ou phrasillons. (348)

Avec mot-phrase, Tesnière propose une lexie spécifique, explicitant la valeur syntaxique, pour désigner une catégorie grammaticale traditionnelle (les interjections) ainsi que d'autres éléments de la langue ou faits de discours (oui, voici par exemple). L'expression permet donc de désigner une catégorie hétérogène de fonctionnement, le néologisme comportant désormais un sens général et un sens particulier :

a) Mot qui forme phrase à lui seul. Si (...) connaît un emploi plein de mot-phrase dans les assertions positives après un énoncé négatif (assertif, jussif ou interrogatif) : Il n'est pas là ? - Si !... il correspond sémantiquement à la phrase c'est ainsi (Moignet, Systématique de la lang. fr., 1981, p.320). b) En partic. Synon. de interjection. Puisque les interjections jouent dans le discours le même rôle que des phrases entières, nous les appellerons des mots-phrases (Tesn. 1959, p.95). Rem. Phrase-mot ds Lar. encyclop.: inusité sous cette forme. (TLF)

En raison de la diversité des unités linguistiques qu'il recouvre, le terme est devenu l'équivalent de marqueur discursif et insert (Kahane et Mazziotta 2015), qui correspondent chacun à l'angle (sémantico-pragmatique ou morphosyntaxique) à partir duquel le fait est envisagé.

La grammaire traditionnelle ne traite pas vraiment le problème des mots-phrases (Maziotta et Kahane 2016), pas plus que Tesnière ne les intègre finalement à sa syntaxe. La conception de la phrase comme unité syntaxique, ilôt de dépendances rectionnelles, se heurte en français parlé, mais aussi dans les genres écrits, au problème des mots-phrases, qui remettent en cause le concept même de phrase comme unité définie par une organisation interne. Le néologisme mot-phrase a le mérite de faire saillir - à défaut de la résoudre - cette problématique.

Tesnière a créé un autre néologisme synonyme de mot-phrase, qui s'appuie sur un procédé original dans le métalangage, une double suffixation diminutive : le suffixe -ille, qui représente le latin -icula, formateur de substantifs féminins, et le suffixe -on, qui forme de nombreux substantifs souvent masculins et concrets (raidillon, oisillon, etc.). Dans le contexte de la linguistique, le terme pourrait avoir une connotation péjorative, ce que Jack Feuillet conteste en partie :

Le seul qui ait entrepris de creuser un peu plus les choses et Tesnière [,] qui consacre un chapitre aux «phrasillons ». On notera à ce propos que le suffixe -illon, qui n'es peut-être pas péjoratif dans l'esprit de Tesnière, suggère néanmoins le manque de considération, confirmée par le fait qu'on s'en débarrasse assez vite (car ils se prêtent mal à une classification structurale) et qu'il ne les compte pas dans ce qu'il appelle les « espèces de phrases ». (Feuillet 1988 : 110 ; cité dans Mazziotta et Kahane 2016)

Un tel mode de formation trahit en tout cas l'imaginaire du linguiste, car chez Tesnière, phrasillon apparaît superfétatoire du fait qu'il est donné comme synonyme d'un autre néologisme. La redondance terminologique procède alors peut-être aussi de la difficulté à intégrer ces objets dans la classification grammaticale.

\subsubsection{Sous-phrase et phrase-noyau}

Au début du XX $X^{\mathrm{e}}$ siècle, Damourette et Pichon (1911-1927 : 128) emploient déjà l'unité lexicale sous-phrase explicitement comme synonyme de proposition subordonnée, dans la terminologie grammaticale traditionnelle, héritée de la conception logique de la grammaire générale du XVII ${ }^{\mathrm{e}}$ siècle. Elle a été reprise par la linguistique structurale et générative, ainsi de 
manière consistante, «[1]a linguistique actuelle, par une sorte de synecdoque, appelle parfois phrase la proposition principale, les subordonnées étant appelées sous-phrases (Dupré 1972) » (cité dans TLF).

Le nouveau terme permet principalement d'éliminer la notion logique périmée de proposition (Léon 2003, Combettes 2011). De plus, il renvoie à la critique de la notion de subordination dans le cadre des «propositions », et permet d'écarter toute ambiguïté en matière d'extension de la notion de subordination en grammaire. Une expression problématique de la grammaire traditionnelle se voit ainsi supplantée, mais le gain conceptuel reste limité puisque l'idée de hiérarchie persiste dans la métaphore spatiale sous-jacente.

Un troisième mot composé, phrase-noyau iii, émerge dans les années 1960. Il fait l'objet d'une entrée dans le Dictionnaire de linguistique de Dubois et al. (1994) :

1. En grammaire structurale, la phrase-noyau est la phrase déclarative active transitive réduite à ses constituants fondamentaux. L'enfant lit un livre est une phrase-noyau. 2. En grammaire générative. syn. de phrase nucléaire*.

Ce terme est donc l'antonyme de sous-phrase, au plan morphologique (eu égard à la distribution de ses lexèmes) comme au plan sémantique. La dimension métaphorique est cette fois évidente, éclairant rétrospectivement dans ce sens le terme sousphrase. La métaphore témoigne d'une démarche heuristique visant à se défaire de l'approche hiérarchique très marquée par l'histoire de la grammaire.

Ces lexies touchent à des notions problématiques dans la définition même du concept de phrase. Outre qu'ils disqualifient la notion de proposition, ces deux mots composés sont le lieu d'une expression analogique, comme en témoigne encore le néologisme de Tesnière mot-phrase, fondé sur une relation d'équivalence. La communauté du mode de formation indique un semblable ancrage théorique, le structuralisme, dont le procédé de création lexicologique donne précisément une image qui remotive partiellement les deux signes.

\subsection{La linguistique phraséologique}

La majorité des néologismes du siècle dernier sont des dérivés suffixaux qui ne sont pas (encore) enregistrés dans les dictionnaires de langue - ni même dans les dictionnaires de spécialité ${ }^{\text {iv }}$-, mais dont on observe l'emploi dans la littérature scientifique.

En 1909, dans son Traité de stylistique française, Charles Bally s'intéressait à ce qu'il nommait de manière périphrastique les « unités phraséologiques ». Au moins deux néologismes concurrents se sont implantés au cours de ces dernières décennies, pour désigner les «tournures typiques d'une langue, soit par leur fréquence, soit par leur caractère idiomatique » qui constituent l'ensemble de la phraséologie : phrasème et phraséologisme, voire phraséoterme (Gautier 2002) - plus rare - présentent l'intérêt d'être synthétiques. À l'instar de mot-phrase, c'est le problème de la frontière entre lexique et syntaxe qui est visé par ces néologismes. En effet, ils désignent à proprement parler des unités syntagmatiques, et non des unités phrastiques, se substituant ainsi au signe phrase dans son emploi à l'époque classique.

Phrasème est formé sur le modèle des noms désignant les unités linguistiques distinctives (phonème, graphème, lexème, etc.), c'est-à-dire avec le suffixe -ème. En dépit de son allure scientifique, le terme est pour le moins paradoxal dans la mesure où la notion de phrase n'est pas en jeu dans son signifié. Benveniste emploie ce dernier terme en 1966 dans Problèmes de linguistique générale I, mais avec un sens étymologique : « La phrase n'est pas une classe formelle qui aurait pour unités des 'phrasèmes ' délimités et opposables entre eux » (p. 129). Le sens conforme à son mode de formation, qui plaiderait en faveur de l'existence d'unités distinctives de la phrase, se voit ainsi récusé, et le terme présenté comme fictif. Il est actuellement utilisé de manière courante, en linguistique, au sens d'unité phraséologique, témoignant à la fois d'un affaiblissement et d'une spécialisation sémantiques.

En revanche, le terme phraséologisme apparaît parfaitement clair et cohérent avec sa base (phraséologie) du point de vue sémantique, permettant d'écarter toute ambiguité, ce qui explique probablement son émergence.

Dubois et alii (1994 : 366) pointent la difficulté qu'il y a à délimiter le champ des phraséologismes :

Un dictionnaire phraséologique s'assigne pour un objet le recensement et la présentation des expressions figées spécifiques à une langue. En principe, il n'enregistre pas les proverbes, pour autant que ceux-ci représentent des unités phrastiques complètes. Il devra également exclure le cliché de son champ d'étude : à la différence du cliché, écart stylistique banalisé par la répétition (l'astre des nuits, des doigts de fée), la phraséologie se définit non par l'écart qu'elle représente par rapport à la langue, mais par le caractère stabilisé de la combinaison qu'elle constitue. Un dictionnaire phraséologique n'est qu'un sous-ensemble particulier d'un dictionnaire syntagmatique, qui envisagerait les types de combinaisons les plus courants d'une langue dans une synchronie donnée.

Si la discipline émerge dans les années 1960, les travaux pionniers de la linguistique phraséologique datent des années 1980. Les recherches dans ce domaine sont actuellement abondantes ${ }^{v}$ en raison du stock très élevé des unités phraséologiques de la langue. Trois critères permettent de les définir: la polylexicalité, le figement et la noncompositionnalité (ou opacité) du sens. Le terme phraséologie a donc au moins partiellement perdu le lien avec son radical, puisqu'il désigne des unités lexicales : 
(...) ensemble des unités complexes du lexique, qui présentent des degrés variables de figement, qui sont construites dans des contextes spécifiques, et qui sont tenues à cet égard pour caractéristiques d'un type de discours. (Neveu $2004: 231$ )

Il s'agit désormais d'un mot-concept.

La marginalisation, voire l'élimination du sens propre a eu pour conséquence le recentrage sur les unités lexicales complexes au détriment de la phrase conçue comme unité syntaxique. Cette réduction à un niveau inférieur de la langue se voit pourtant aujourd'hui contestée, d'aucuns plaidant pour l'élargissement des études phraséologiques à la phrase (Legallois et Tutin 2013).

L'expression «Raison phrastique »-allusion au texte philosophique de Kant, Critique de la raison pratique (1788), dans l'approche épistémologique d'Antoine Gautier (2006) - cristallise le statut du concept de phrase dans la linguistique de la seconde moitié du XXe siècle. Dans le cadre de l'effort théorique en faveur de la délimitation de la notion et de sa réduction à une catégorie stabilisée, le dérivé phrasticité émerge encore sous la plume épistémologique du même A. Gautier (2006 : 59), ainsi que dans divers articles portant sur les langues du monde ${ }^{1}$.

\section{Conclusion}

Phrase est un mot-morphème productif du XVIIe au $\mathrm{XX}^{\mathrm{e}}$ siècle, selon les principales voies de la création lexicale que sont l'emprunt, la dérivation suffixale et la composition populaire, qui se répartissent de manière cohérente selon les époques et les ancrages disciplinaires. L'évolution du champ morphologique permet ainsi de suivre la circulation de la notion de phrase depuis la rhétorique jusqu'aux différentes approches linguistiques modernes, où la créativité lexicale est adossée à un effort de théorisation sans précédent de la notion de phrase.

Globalement, les définitions de la phrase attestées dans les dictionnaires depuis le XVIIe siècle emportent avec elles des connotations souvent négatives, que l'imagination onomasiologique a spécifiées dans la création de dérivés morphologiques. La linguistique a permis, au XXe et XXIe siècles, la conversion progressive de ces valeurs négatives en éléments d'une nouvelle terminologie intégrée à des cadres théoriques définis.

Si la diversification terminologique à l'époque moderne s'efforce de traiter une partie des problèmes posés par la notion de phrase, plutôt que de proposer des sous-spécifications conceptuelles, certains néologismes de forme créent des doublons dans un même cadre théorique. Cet excès d'étiquetage et de technicité peut témoigner d'une recherche d'ajustement terminologique, les innovations lexicales se présentant comme des tentatives de (re)conceptualisation de notions anciennes devenues caduques, ou d'une notion épineuse. Les néologismes peuvent également constituer une manifestation de la concurrence dans le champ d'une linguistique professionnalisée.

À l'époque contemporaine, le discours métalinguistique spontané n'échappe pas non plus totalement à l'innovation lexicale, ainsi que l'atteste la création toute récente de phrasitude, calque du latin, selon la résurgence d'une suffixation philosophique accordée à l'époque contemporaine (Khoel 2012), ou mot-valise (phrase + attitude), en liaison avec la popularité de la locution petite phrase qui opère un retour des locutions (péjoratives) attachées à l'époque classique et au $\mathrm{XIX}^{\mathrm{e}}$ siècle - mais dans le cadre revalorisé de la phraséologie.

\footnotetext{
${ }^{1}$ Jocelyne Fernandez-Vest (2008), «Subordination et degrés de phrasticité dans quelques langues ouraliennes : exemples samiques et fenniques », La Linguistique 2008/2 (Vol. 44), 99-116 ; Outi Duvallon (2016), «Individuation référentielle de la construction infinitive V-TESSA en finnois : entre détermination nominale et complémentation verbale ", dans Antoine Gautier, Eva Havu, Dan Van Raemdonck, (dirs), DéterminationS, P.I.E Peter Lang, 978-2-87574-326. 〈halshs-01341838〉
} 


\section{Références bibliographiques}

Académie française (1694-1878). Dictionnaire de l'Académie française. $1^{\text {ère }}$ éd. 1694, Vve de J.-B. Coignard et J.-B. Coignard ; 2e éd. 1718, J.-B. Coignard ; $3^{\mathrm{e}}$ éd 1740, J.-B. Coignard ; $4^{\mathrm{e}}$ éd. 1762, Vve Brunet ; $5^{\mathrm{e}}$ éd. 1798, J. Smits \& Co ; $6^{\mathrm{e}}$ éd. 1835, Firmin-Didot Frères ; $7^{\mathrm{e}}$ éd. 1878, Firmin-Didot \& Cie.

Albalat, A. (1899). L'Art d'écrire enseigné en vingt leçons. Paris : Armand Colin.

Bès, G. (1999). La phrase verbale noyau en français. Recherches sur le français parlé 15, 237-358; https://hal.archives-ouvertes.fr/hal01005527/file/1999_La_phrase_verbale_noyau.pdf.

Bally, C. (1909). Traité de stylistique. Genève : Georg ; Paris : Klincksieck.

Combettes, B. (2011). Phrase et proposition. Histoire et évolution de deux notions grammaticales. Le Français aujourd'hui 173, 11-20 ; https://www.cairn.info/revue-le-francais-aujourd-hui-2011-2-page-11.htm.

Cormenin, L. de - (1841). Livre des Orateurs. Paris: Pagnerre.

Damourette, J. et Pichon, E. (1911-1927). Essai de grammaire de la langue française: des mots à la pensée t. I. Paris: D’Artrey; https://gallica.bnf.fr/ark:/12148/bpt6k62820045.texteImage.

Deschanel, É. (1898). Les Déformations de la langue française. Paris : Calmann Lévy.

Diderot, D. Le Rond d'Alembert, J. (1751-1772). Encyclopédie, Dictionnaire raisonné des Sciences et des Arts. Neufchastel : Samuel Faulche $\&$ Cie.

Dubois, J. et al. (1994). Dictionnaire de linguistique et de sciences du langage. Paris : Larousse.

Duvallon, Outi (2016). Individuation référentielle de la construction infinitive V-TESSA en finnois : entre détermination nominale et complémentation verbale. A. Gautier, E. Havu et D. Van Raemdonck, (dirs), DéterminationS. Bruxelles : Peter Lang, 978-2 574326. 〈halshs-01341838>

Fenoglio, I. (2019). Proposition, phrase, énoncé chez Émile Benveniste. F. Neveu (dir.), Proposition, phrase, énoncé. Linguistique et philosophie. Paris : éditions ISTE, 183-203.

Féraud, J. F., Abbé (1761). Dictionnaire grammatical de la langue française. Avignon : Vve Girard.

Féraud, J. F., Abbé (1788). Dictionnaire critique de la langue française t. 3. Marseille : Mossy.

Fernandez-Vest, Jocelyne (2008). Subordination et degrés de phrasticité dans quelques langues ouraliennes : exemples samiques et fenniques. La Linguistique 2008/2 (Vol. 44), 99-116

Fournier, Nathalie (1998). Grammaire du français classique. Paris : Belin.

Gautier, A. (2006). Unité et discontinuité : une approche épistémologique et systématique de la phrase. Thèse de Doctorat, Université ParisSorbonne.

Gautier, L. (2002). Terme, phraséoterme, phrasème : questions de délimitation en langue spécialisée. Le Continuum en linguistique. Sousse : Tunisie, 153-172; https://hal.archives-ouvertes.fr/hal-00201271/document.

Grevisse, M. (1936). Le Bon usage. Bruxelles : Duculot.

Haßler, G. (2012). La linéarité du langage comme problème théorique dans les théories rationalistes et sensualistes. Beiträge zur Geschichte der Sprachwissenschaft 22.1,33-66.

Kahane, S., et Mazziotta, N. (2015). Quel classement syntaxique pour les 'marqueurs discursifs', 'mots-phrases' et autres 'inserts' ? Prédicatifs et locutifs. Travaux de linguistique 71, 7-42; https://www.cairn.info/revue-travaux-de-linguistique-2015-2-page-7.htm.

Koehl, A. (2012). Altitude, négritude, bravitude ou la résurgence d'une suffixation. $3^{e}$ Congrès Mondial de Linguistique Française. Lyon, 1307-1327.

Larousse, P.-A. (1863-1873). Grand Dictionnaire Universel du XIXe siècle t. 14. Paris : Larousse.

Legallois, D. et Tutin, A. (2013). Vers une extension du domaine de la phraséologie. Langages 189, 3-29; https://www.cairn.info/revuelangages-2013-1-page-3.htm.

Léon, J. (2003). Proposition, phrase, énoncé dans la grammaire: Parcours historique. L'Information grammaticale 98, 5-16; https://www.persee.fr/doc/igram_0222-9838_2003_num_98_1_2610.

Littré, É. (1863-1872). Dictionnaire de la langue française. Paris : Hachette.

Mazziotta, N. et Kahane, S. (2016). Le mot-phrase dans les conceptions de Lucien Tesnière. Bulletin de la Société de Linguistique de Paris 111, 71-107; https://kahanedotfr.files.wordpress.com/2017/01/locutif-bslp2016.pdf.

Melis, L. et Desmet, P. (2000). La phrase et son analyse. Modèles linguistiques 42, 79-145.

Neveu, F. (2004). Dictionnaire encyclopédique des sciences du langage. Paris : Armand Colin.

Neveu, F. (dir.) (2019). Proposition Phrase Énoncé. Londres : ISTE Éditions.

Raby, V. (2002). L'Analyse de la phrase complexe dans la Grammaire Générale. Histoire Épistémologie Langage XXIV 1, 93-105.

Raby, V. (2008) en collaboration avec J.-M. Fournier. Faire une histoire de la grammaire française aujourd'hui faits, hypothèses, propositions. Durand J., Habert B., Laks B. (éds.), Congrès Mondial de Linguistique Française. Paris, Institut de Linguistique Française, 957-969.

Raby, V. (2018). Les Théories de l'énoncé dans la Grammaire générale. Lyon : ENS Éditions.

Seguin, J.-P. (1993). L'Invention de la phrase au XVIIIe siècle : contribution à l'histoire du sentiment linguistique français. Louvain-laNeuve : Bibliothèque de l'Information grammaticale, Éditions Peeters.

Tesnière, L. (1936). Sur la classification des interjections. A. Sestak et A. Dokoupil (dirs.), Mélanges dédiés à la mémoire de Prokop M. Haskovec par ses amis et élèves. Brno : Globus, 343-352.

Vaupot, S. (2012). Les critères de (non-)maintien du néologisme par dérivation dans l'œuvre de Louis-Sébastien Mercier. Zeitschrift für romanische Philologie 128, 474-491.

Wilmet, M. (1997). Grammaire critique du français. Paris : Hachette ; Louvain-la-Neuve : Duculot. 
${ }^{\mathrm{i}}$ Larousse a cité auparavant Montaigne, Fénelon.

${ }^{i i}$ En effet cette création lexicale est imputée à Simon-Nicolas-Henri Linguet (1736-1794), cf. Annales politiques, civiles et littéraires du dix-huitième siècle (1777-1792, t. XV, 45), mais a été oubliée depuis par tous les dictionnaires consultables.

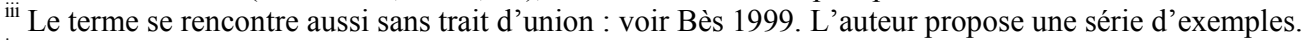

${ }^{\text {iv }}$ Le premier bénéficie néanmoins d'une entrée dans le Wiktionnaire, le second dans Wikipédia.

${ }^{v}$ Comme en témoigne le nombre des colloques internationaux qui ont eu lieu ces cinq dernières années : « Europhras 2014, Europäische gesellschaft für phraseologie », 10-12 septembre 2014, Paris Sorbonne ; «Phraséologie française », Université d’Artois, 21-22 septembre 2017 ; «La phraséologie française : sens, cotextes, contextes », 24-25 mai 2018, Université Marie Curie-Sklodowska de Lublin (Pologne) ; colloque PhraseoRom, 13-15 mars 2019, Friedrich-Alexander-Universitat Erlangen-Nürnberg (Allemagne). Ainsi que celui annoncé à l'Université de Sherbrooke, du 4 au 8 mai 2020 : «Les phraséologismes pragmatiques : Préfabrication et lexiculture ». Etc. 